\title{
Impact of coronavirus disease 2019 on professional practices of audiologists and speech-language pathologists in India: A knowledge, attitude and practices survey
}

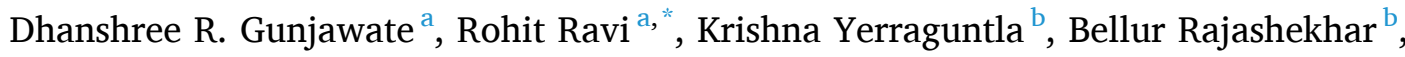 \\ Ashwani Verma ${ }^{\mathrm{c}}$ \\ ${ }^{a}$ Department of Audiology and Speech Language Pathology, Kasturba Medical College, Mangalore, Manipal Academy of Higher Education, India \\ ${ }^{\mathrm{b}}$ Department of Speech and Hearing, Manipal College of Health Professions, Manipal Academy of Higher Education, India \\ ${ }^{\mathrm{c}}$ The Campbell Collaboration, New Delhi, India
}

\section{A R T I C L E I N F O}

\section{Keywords:}

Coronavirus disease 2019

COVID-19

audiologists

speech-language pathologists

Survey

\begin{abstract}
A B S T R A C T
Background: Coronavirus disease 2019 (COVID-19) has spread throughout the world and become a global pandemic. This has hampered and led to drastic changes in the functioning of healthcare services, forcing the professionals to adapt and work efficiently. The present study aimed to explore the impact of COVID-19 on the professional practices of audiologists and speech-language pathologists in India using a cross-sectional knowledge, attitude and practices survey.

Material and methods: The study was conducted in two phases; phase one involved development and validation of the questionnaire, while phase II involved data collection. A cross-sectional self-reported internet-based study using convenience sampling was carried out.

Results: Two hundred and eleven audiologists and speech-language pathologists responded to the survey. Overall, the professionals exhibited good knowledge levels regarding the COVID-19 outbreak. However, there were differences in their attitudes towards service delivery in the midst of the pandemic. Further, poor practices towards infection control measures especially in terms of hand washing was noted.

Conclusion: The findings of the present study are useful in highlighting the need to create better awareness among these professionals about appropriate and standard infection control measures. There is a need to have in place standard operating protocols for hand wash and infection control as well as inclusion in curriculum.
\end{abstract}

\section{Introduction}

A local outbreak of pneumonia of an initially unknown origin was detected in Wuhan (Hubei, China). This was determined to be caused by a novel virus, and subsequently named as severe acute respiratory syndrome coronavirus 2 (SARS-CoV-2) or corona virus disease 2019 (COVID-19). ${ }^{1}$ In the initial few days, maximum cases were reported from different provinces of Mainland China. On $13^{\text {th }}$ January 2020, the first case of COVID-19 was reported outside China in Thailand and by $23^{\text {rd }}$ January 2020, cases were reported from Republic of Korea, Thailand, Japan and Singapore. As the cases started increasing, the World Health Organization (WHO) declared the outbreak of COVID-19, a public health emergency of international concern on $30^{\text {th }}$ January 2020. By $7^{\text {th }}$ March 2020, the number touched $1,00,000$. On $11^{\text {th }}$ March 2020, WHO made a declaration that the COVID-19 outbreak can be categorized as a pandemic. A pandemic is an epidemic disease that is spread across the world. As of $24^{\text {th }}$ March 2020, the outbreak was reported from as many as 177 countries with more than $3,34,981$ confirmed cases. ${ }^{2}$

In India, the first case of positive COVID-19 was reported on $31^{\text {st }}$ January 2020, with a recent travel history from China. Subsequently, by $3^{\text {rd }}$ February 2020, a total of 3 positive cases were reported who were treated and discharged. The situation report update dated $28^{\text {th }}$ February 2020 indicated no fresh cases. However, by 9 th March, 9 cases were reported and the number escalated to 360 by 22 nd March $2020 .^{3}$ The government issued several orders such as enforcement of social distancing, urging people to stay at home barring essential services, travel restrictions, and importance towards hand hygiene. ${ }^{4}$ The sudden increase in the positive COVID-19 cases with changing orders and

\footnotetext{
* Corresponding author.

E-mail address: rohitravi94@gmail.com (R. Ravi).
} 
increasing restrictions led to panic and apprehension among the citizens. However, professionals involved in patient care and rehabilitation services continued to work and provide services.

One such professional involved in providing rehabilitation services are audiologists and speech -language pathologists. As members of health care, these professionals are expected to extend their services to patients and caregivers/bystanders exhibiting symptoms of COVID-19. Their patient load involves pediatric and geriatric population as well as individuals with speech and hearing disability. Most audiological procedures require a direct patient contact while giving instructions, testing (such as placing headphones, probe tips, otoscope specula, electrodes, microphones, impression syringes, earmolds and hearing aids) as well as during counselling. The testing is conducted in a sound treated room or an enclosed chamber with no ventilation. Speech therapy requires an emphasis on mouth/lip expressions (such as in articulation therapy) which would be ineffective wearing a mask. Certain procedures such as Fiberoptic Endoscopic Evaluation of Swallowing (FFES) and laryngoscopy involve the nose and nasopharynx. Procedures such as FEES involve using a spray that could aerosolize pathogens on the mucosa. In general, the risk of using instruments and the contact with patients and caregivers/bystanders cannot be overlooked. Further, the absence of formal training in infection control measures as a part of these professionals' curriculum could increase the chances of them missing important steps in infection control and leading to contracting the virus.

The WHO, ${ }^{5}$ Centre for Disease control and Prevention ${ }^{6}$ and government authorities ${ }^{7}$ have provided support, guidelines and advisories on the COVID-19 pandemic. The professional bodies for audiologists and speech language pathologists have also made available relevant educational materials and guidelines for professional practices. ${ }^{8-10}$ However, lot of myths and misinformation have been observed. Thus, it becomes relevant to know the impact of this outbreak and its associated symptoms on patient care and service delivery among audiologists and speech- language pathologists. The present study was conducted with an aim to study the impact of COVID-19 on the professional practices of audiologists and speech- language pathologists in India using a cross-sectional knowledge, attitude and practices (KAP) survey.

\section{Method}

The study was in accordance with the Helsinki declaration. ${ }^{11}$ The institutional ethics committee permitted online surveys on COVID-19 during the lockdown period when this study was conducted. All participants were explained about the purpose of the study. The participation was voluntary. The present study was conducted in two phases; phase I involved the development and validation of the questionnaire, while phase II, data collection.

\subsection{Phase I: Development and validation of the questionnaire}

A self-reported questionnaire was used to gather data on the impact of COVID-19 on professional practices of audiologists and speechlanguage pathologists working in India. This questionnaire was developed based on the information about COVID-19 on official websites of World Health Organization ${ }^{2}$; Centers for Disease Control and Prevention, ${ }^{6}$ American Speech-Language-Hearing Association, ${ }^{9}$ Ministry of Health and Family Welfare, Government of India ${ }^{4}$ and discussion with subject experts. One of the authors has also completed specific courses on infection prevention and control for COVID-19 by OpenWHO. The developed questionnaire was content validated by three audiologists and speech-language pathologists as well as three public health experts, all with a minimum of 5 years of research and work experience. Every expert rated each question using a rating scale of irrelevant, somewhat relevant, quite relevant and relevant. The items rated relevant and quiet relevant were included in the final questionnaire. A score of 0.9 was obtained on the content validity index for Scale (S-CVI) for the final questionnaire, indicative of excellent content validity (Polit \& Beck, 2006). All the questions proposed in the initial version were rated to be of relevance and hence retained in the final questionnaire. The questionnaire comprising of 23 items; demographic details, knowledge and attitudes towards COVID-19, and practices related to infection control. These questions included a blend of true/false, yes/no, multiple-choice questions, checkboxes and short answer types.

\subsection{Phase II: Data collection and analysis}

A cross-sectional self-reported internet-based study design using convenience sampling was implemented for the present study.

\subsection{Data collection}

The finalized questionnaire was made available using Google Form, with an email link for the same. Five hundred audiologists and speechlanguage pathologists were randomly chosen from the Indian SpeechLanguage and Hearing Association members list. The questionnaire link was shared with these professionals through their personal email ids and messaging service such as WhatsApp. No personal information was collected to maintain anonymity. All responses were saved on automatically with access only to the primary author.

The data collection was carried out from 15th to $21^{\text {st }}$ March 2020. The questionnaire was administered during the second stage of the COVID-19 spread and before the complete shutdown of nonessential/ non-emergency services. The Google form started with a brief description of the present study followed by a formal consent statement. Only those participants who consented to participate could proceed further. The questionnaire was administered in English and took approximately $10 \mathrm{~min}$ for completion.

\subsection{Data analysis}

The data analysis was done using descriptive statistics comprising of mean and SD for continues variables and frequency and percentage for discrete variables. All analysis was done using SPSS 15.

\section{Results}

Two hundred and eleven audiologists and speech-language pathologists practising in India responded to the survey. All the questions were compulsory in order to submit the Google form; hence there were no incomplete responses. Table 1 depicts the demographic details of the professionals who participated in the study.

\subsection{Knowledge of symptoms of COVID-19 and its preventive measures}

After the questions on demographic information, the questionnaire comprised of seven questions related to knowledge of symptoms of COVID-19 and its preventive measures. Out of the seven, four questions

Table 1

Demographic details of the participants.

\begin{tabular}{llll}
\hline & & Mean \pm SD & Range \\
\hline Age (in years) & & $29.80 \pm 9.32$ & 21 to 69 \\
Experience (in years) & & $6.99 \pm 8.30$ & $1-40$ \\
& & Number (Percentage) \\
Gender & Male & $61(28.6 \%)$ \\
& Female & $149(70 \%)$ \\
Highest qualification & Prefer not to say & $1(0.5 \%)$ \\
& Bachelors & $72(34.1 \%)$ \\
& Masters & $122(57.8 \%)$ \\
Work setting & Doctorate & $17(8.1 \%)$ \\
& Private practice & $69(32.7 \%)$ \\
& Hospital & $66(31.3 \%)$ \\
& Teaching and clinical services & $76(36 \%)$ \\
\hline
\end{tabular}


were true-false-not sure statements while the remaining were multiplechoice options. $93.8 \%$ of respondents correctly identified the core symptoms of COVID-19 such as fever, tiredness and dry cough, while $6.2 \%$, could not. The correct response for the statement; 'COVID-19 patients also develop aches, pain, nasal congestion, runny nose, sore throat or diarrhea', should have been true. $66.4 \%$ responded as true; remaining $17.1 \%$ responded false while $16.6 \%$ were not sure. Next, 'COVID-19 infection can spread through small droplets from nose or mouth of infected person' was a true statement. A majority (93.4\%) responded correctly, while $6.6 \%$ were incorrect. The fourth statement 'wearing multiple masks, smoking and consuming alcohol are effective against COVID-19' was a false statement for which $82 \%$ responded correctly. Surprisingly, $10.4 \%$ were unsure, and $7.6 \%$ reported that these measures are effective.

The remaining three questions under the knowledge domain were related to ideal distance to be maintained, incubation period, and recommended composition of the hand sanitizers. The ideal distance to be maintained from a person with COVID-19 infection is 3 feet. This was correct for only $50 \%$ of the respondents $(105,49.8 \%)$. The remaining responded incorrectly,viz. 1 feet $(15.2 \%), 6$ feet $(28 \%), 8$ feet $(4.3 \%)$ and not sure $(2.3 \%)$. Majority $(80.6 \%)$ of the professionals responded correctly for the question on the incubation period (the period between contacting virus and showing symptoms) of 1-14 days. The remaining responses included; $15-20$ days (16.1\%), $21-25$ days (1.9\%) and beyond 25 days $(1.4 \%)$. The final question under the knowledge domain was based on the recommended composition of the hand sanitizers for which $60-95 \%$ alcohol was the expected answer. However, less than $50 \%$ (48.8\%) replied correctly that the composition should include 60-95\% alcohol. The remaining responses were distributed across other options; $20-50 \%$ alcohol (25.6\%), 0-10\% alcohol (21.3\%) and 100\% alcohol $(4.3 \%)$.

\subsection{Attitudes towards COVID-19 infection}

The next five questions were aimed at exploring the attitudes towards COVID-19 infection. The response categories included strongly disagree, disagree, neutral, agree and strongly agree. Table 2 displays the frequency and percentages of responses for these statements.

\subsection{Practices towards infection control and sources of information}

Eleven questions were based on practices and infection control measures implemented to curtail the spread of COVID-19. The participants were asked about the different preventive measures they use while imparting clinical services. These included the use of masks, hand sanitizers, gloves, washing hands, and maintaining appropriate distance. The responses are depicted in Fig. 1.

The next four questions were based on the current changes in practices followed by the clinicians in terms of patient care. The respondents were asked to report the measures they would incorporate if they were to develop any symptoms related to COVID-19. More than 90\% (91.9\%) expressed that they would get screened or tested. The remaining responses included waiting for some time for symptoms to resolve (3.8\%) and taking home remedies (4.3\%). The same positive practices were also applicable for any of their patients exhibiting symptoms related to COVID-19. 72\% responded that they would recommend medical advice immediately while $21.3 \%$ would recommend their patient to discontinue therapy and return when symptoms resolved. Only $6.2 \%$ responded that they would discharge their patient to ensure their own safety while $0.5 \%$ to suggest remedies on their own.

\subsection{Based on the responses, most of the participants would practice preventive measures related to travel as noted in Table 3}

Maximum number of participants were using hand wash procedures (82.9\%) while $17.1 \%$ were not following it. Among the ones who used
Table 2

Attitudes towards COVID-19.

\begin{tabular}{|c|c|c|c|c|c|}
\hline & $\begin{array}{l}\text { Strongly } \\
\text { disagree n } \\
(\%)\end{array}$ & $\begin{array}{l}\text { Disagree } \\
\mathrm{n}(\%)\end{array}$ & $\begin{array}{l}\text { Neutral } \\
\text { n (\%) }\end{array}$ & $\begin{array}{l}\text { Agree n } \\
(\%)\end{array}$ & $\begin{array}{l}\text { Strongly } \\
\text { agree n } \\
(\%)\end{array}$ \\
\hline $\begin{array}{l}\text { I would continue } \\
\text { to provide my } \\
\text { services, even if } \\
\text { there is a } \\
\text { COVID-19 } \\
\text { positive case in } \\
\text { my hospital/ } \\
\text { vicinity. }\end{array}$ & $\begin{array}{l}70 \\
(33.2 \%)\end{array}$ & $59(28 \%)$ & $\begin{array}{l}43 \\
(20.4 \%)\end{array}$ & $\begin{array}{l}22 \\
(10.4 \%)\end{array}$ & $\begin{array}{l}17 \\
(18.1 \%)\end{array}$ \\
\hline $\begin{array}{l}\text { I would provide } \\
\text { my services to a } \\
\text { patient } \\
\text { exhibiting } \\
\text { symptoms of } \\
\text { COVID-19 }\end{array}$ & $\begin{array}{l}85 \\
(40.3 \%)\end{array}$ & $\begin{array}{l}63 \\
(29.9 \%)\end{array}$ & $\begin{array}{l}27 \\
(12.8 \%)\end{array}$ & $\begin{array}{l}22 \\
(10.4 \%)\end{array}$ & $14(6.6 \%)$ \\
\hline $\begin{array}{l}\text { I am scared of } \\
\text { contacting } \\
\text { COVID-19 from } \\
\text { coming in } \\
\text { contact with } \\
\text { contagious } \\
\text { people in the } \\
\text { workplace }\end{array}$ & $\begin{array}{l}30 \\
(14.2 \%)\end{array}$ & $\begin{array}{l}32 \\
(15.2 \%)\end{array}$ & $\begin{array}{l}46 \\
(21.8 \%)\end{array}$ & $\begin{array}{l}57 \\
(27 \%)\end{array}$ & $\begin{array}{l}46 \\
(21.8 \%)\end{array}$ \\
\hline $\begin{array}{l}\text { I will not permit } \\
\text { my cases to } \\
\text { come for } \\
\text { assessment/ } \\
\text { therapy if they } \\
\text { complain of any } \\
\text { symptoms } \\
\text { related to } \\
\text { COVID-19 }\end{array}$ & $\begin{array}{l}29 \\
(13.7 \%)\end{array}$ & $\begin{array}{l}29 \\
(13.7 \%)\end{array}$ & $\begin{array}{l}18 \\
(8.5 \%)\end{array}$ & $\begin{array}{l}52 \\
(24.6 \%)\end{array}$ & $\begin{array}{l}83 \\
(39.3 \%)\end{array}$ \\
\hline $\begin{array}{l}\text { I feel COVID-19 } \\
\text { will be } \\
\text { successfully } \\
\text { controlled. }\end{array}$ & $18(8.5 \%)$ & $\begin{array}{l}24 \\
(11.4 \%)\end{array}$ & $\begin{array}{l}37 \\
(17.5 \%)\end{array}$ & $\begin{array}{l}72 \\
(34.1 \%)\end{array}$ & $\begin{array}{l}60 \\
(28.4 \%)\end{array}$ \\
\hline
\end{tabular}

hand-washing procedures, only $39.3 \%$ used standard protocols for hand wash. The participants were asked to elaborate upon the infection control procedures being followed while working with patients, depicted in Fig. 2.

When asked whether the professionals would like to shift to telemode for imparting audiological and speech therapy services, $77.3 \%$ responded in affirmative, $13.3 \%$ were not sure while remaining $9.5 \%$ responded in negation.

The different sources of information preferred by the participants have been depicted in Fig. 3. As noted from the figure, the most preferred source of information on COVID-19 was World Health Organization website $(70.6 \%)$ followed by internet search $(64 \%)$. The different responses under other options included family members of doctors, information provided at place of work, information sent by Indian Speech-Language and Hearing Association, medical colleagues, word of mouth and WhatsApp messages.

\section{Discussion}

COVID-19 has become a matter of serious concern across the globe where the entire healthcare sector is fighting hard against this pandemic. Audiologists and speech- language pathologists as health care workers are involved in providing services, involving direct contact with patients. This outbreak of COVID-19 has raised concerns as these patients and/or their caregivers might be exhibiting symptoms of the disease or may be carriers themselves. These challenges are further compounded by the absence of formal training in infection control measures as a part of the professionals' curriculum. As a result, most of the knowledge, attitudes and practices are based on their experience or word of mouth information. In the present outbreak, both audiological 


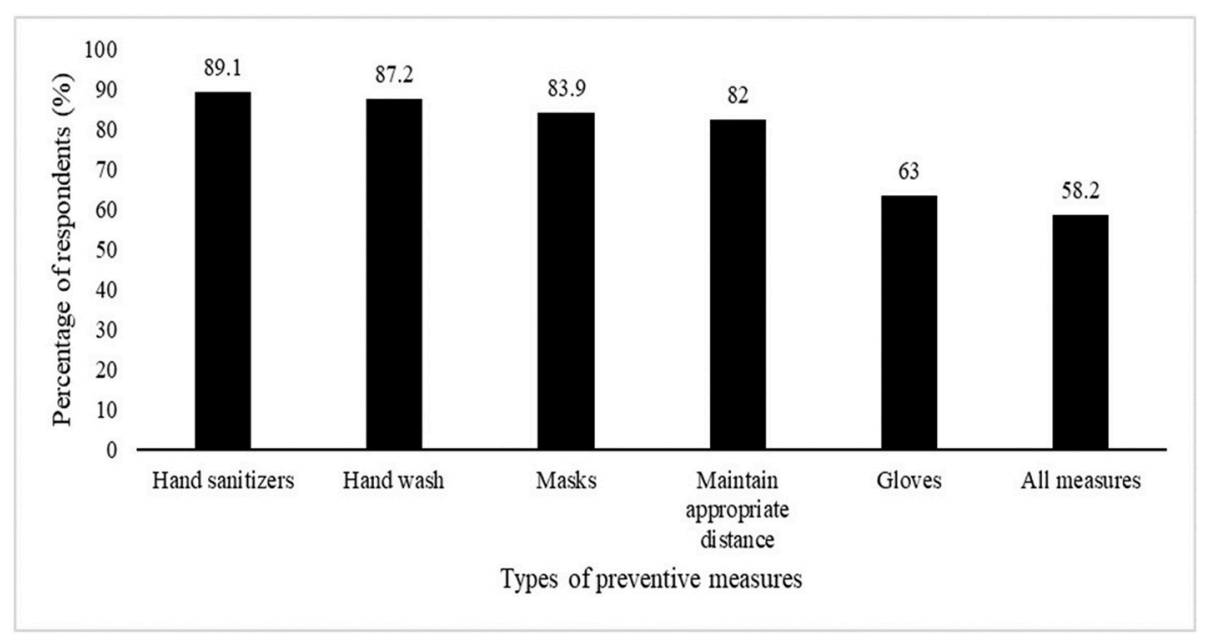

Fig. 1. Types of preventive measures.

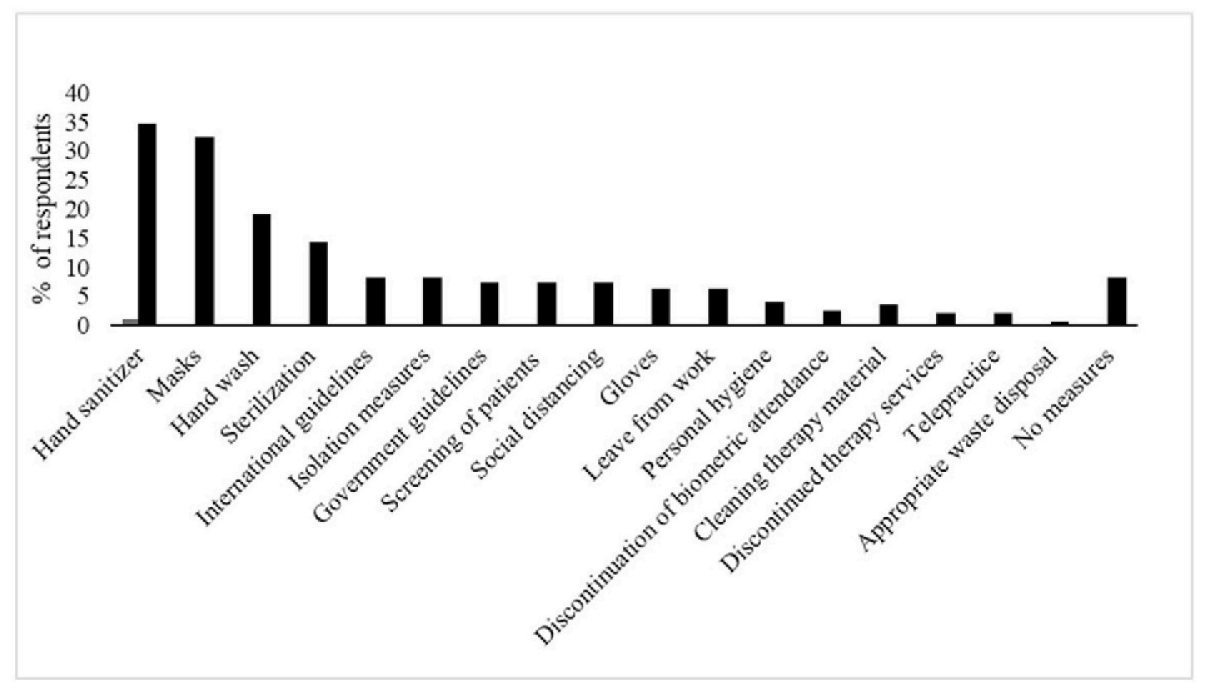

Fig. 2. Infection control procedures being followed.

Table 3

Preventive measures related to travel.

\begin{tabular}{|c|c|c|c|}
\hline & Yes n (\%) & $\begin{array}{l}\text { No n } \\
\text { (\%) }\end{array}$ & $\begin{array}{l}\text { Not sure n } \\
(\%)\end{array}$ \\
\hline $\begin{array}{l}\text { Will you restrict your travel during this } \\
\text { COVID-19 pandemic? }\end{array}$ & $\begin{array}{l}181 \\
(85.8 \%)\end{array}$ & $\begin{array}{l}11 \\
(5.2 \%)\end{array}$ & $19(9 \%)$ \\
\hline $\begin{array}{l}\text { If you travel from an affected area, would } \\
\text { you follow the government guidelines of } \\
\text { isolation? }\end{array}$ & $\begin{array}{l}199 \\
(94.3 \%)\end{array}$ & $\begin{array}{l}3 \\
(1.4 \%)\end{array}$ & $9(4.3 \%)$ \\
\hline $\begin{array}{l}\text { If you have travelled with a suspected person } \\
\text { or infected person from an affected area, } \\
\text { would you follow the government } \\
\text { guidelines of isolation? }\end{array}$ & $\begin{array}{l}204 \\
(96.7 \%)\end{array}$ & $\begin{array}{l}2 \\
(0.9 \%)\end{array}$ & $5(2.4 \%)$ \\
\hline
\end{tabular}

and speech therapy services were available until the closure of nonessential/emergency medical services. It is hence considered crucial to assess the knowledge levels regarding COVID-19, changing attitudes and impact on their practices among these professionals. To the authors' knowledge, this is the first published survey on the knowledge, attitudes, practices towards COVID-19 and sources of information among these professionals.

The professionals who participated in the present study were well distributed across the age range of 21-69 years with 1-40 years of experience. The gender distribution was skewed towards females, with almost $70 \%$ being females. However, this is not surprising as this gender variation in this profession is well established. ${ }^{12}$ Bachelors is the minimum qualification required for practicing in India as an audiologist and speech-language pathologist. Majority of the participants hold a master's degree followed by bachelors and a few were with doctoral degrees. The distribution across the work setting was balanced between clinical, hospital-based as well as academic and clinical settings.

\subsection{Existing knowledge levels of COVID-19}

More than $90 \%$ professionals correctly identified the core symptoms associated with COVID-19 as well as it being transmitted through droplets from nose or mouth. However, the other symptoms such as aches, pain, nasal congestion, running nose, sore throat or diarrhea were less commonly known. This difference could be the result of the emphasis being given towards the core symptoms and mode of transmission across all the awareness drives. As per the guidelines, a distance of 3 feet has been recommended from a person who is coughing or sneezing. ${ }^{4}$ However, only $49 \%$ professionals were able to correctly answer this question. The recommended composition of a hand rub or a hand sanitizer is $60-95 \%$ alcohol $^{13}$; however, this was correctly answered by less than $50 \%$ professionals. 


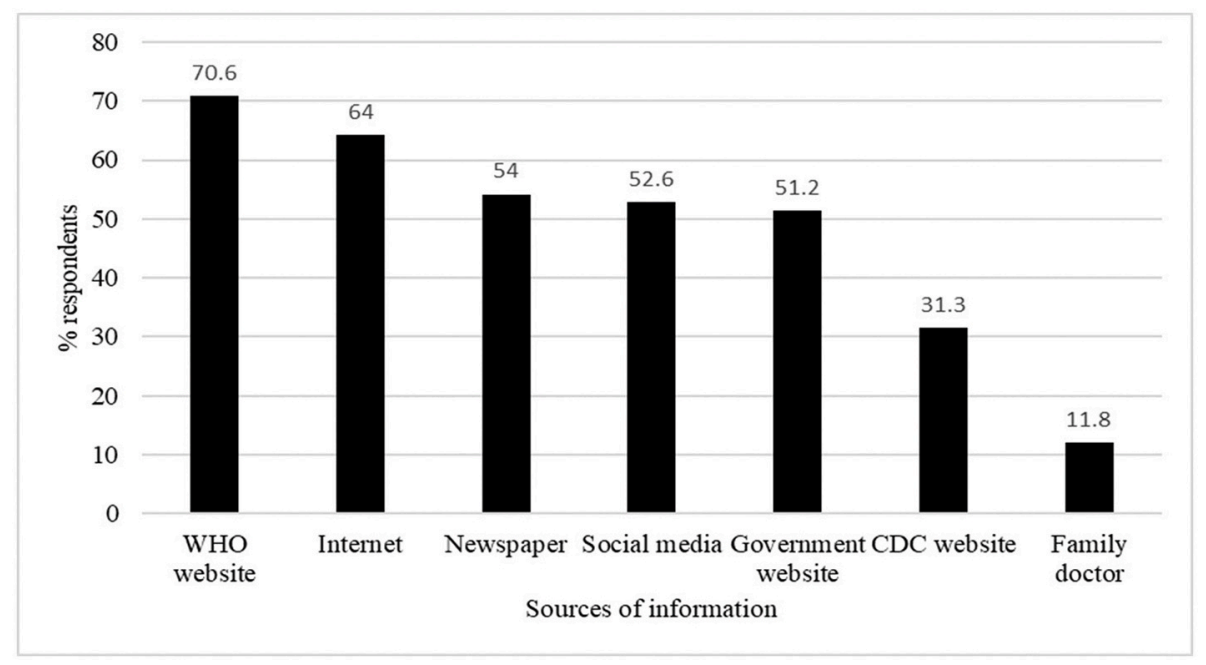

Fig. 3. Sources of information.

\subsection{Attitudes towards COVID-19}

At the time of this survey, the government advisory included only screening of individuals with an international travel history. ${ }^{14}$ However, despite a government advisory and good knowledge levels, these professionals exhibited changes in attitudes towards service delivery for patients. Further, almost $40-60 \%$ of the professionals were reluctant to provide services even to those individuals who were asymptomatic and not confirmed cases. When asked 'I feel COVID-19 will be successfully controlled', half of the participants replied in affirmation, $19 \%$ in negation while $17 \%$ choose to be neutral. These varied responses could be attributed to the partial lockdown of services at the time of the survey. Further, the stress associated with the outbreak and the feeling of being at risk of contracting it might have impacted their attitude towards their service delivery. ${ }^{15}$

\subsection{Practices towards infection control and sources of information}

The professionals were asked to choose the type of preventive measures they would use while imparting clinical services. More than $80 \%$ used sanitizers, hand wash, masks and maintained appropriate distance. However, based on the responses under the knowledge domains, few professionals were aware about the correct composition of the hand sanitizer and appropriate distance to be maintained. Possibly, these practices are the outcome of the awareness drives after the current pandemic outbreak and not based on theoretical knowledge. The professionals exhibited positive practices of getting tested and following government travel guidelines for themselves as well as their patients.

Regular and appropriate hand washing is an essential component of infection control measures and has emerged as a very crucial component in preventing the spread of COVID-19. However, in the present survey, although a large majority of respondents (82.9\%) followed hand wash procedures, only some $(39.3 \%)$ used standard procedures. This attracts attention towards the absence of awareness towards the standard hand washing procedures which has also been observed in other healthcare providers. $^{16}$

Standard infection control precautions emphasize on hand hygiene, isolation, sterilization of reusable instruments, needle safety, wearing personal protective equipment, disposal and waste management. ${ }^{9}$ Based on the responses, it can be noted that most of the respondents followed the precautions at personal level as well as at place of work. Social distancing is the most recommended precautionary measure for preventing COVID-19; however, this was followed by only $7 \%$ of the professionals. Surprisingly, $8 \%$ respondents were not following any infection control measures. Maximum respondents preferred reliable sources of information on COVID-19 such as World Health Organization website. Nevertheless, a considerable number also reported the use of internet and social media. The validity of information from internet and social media is questionable.

\section{Conclusion}

The present survey was conducted using a self-rated questionnaire and hence assessing the real practices was beyond the scope of this study. Presently, India is slowly lifting the lockdown and restrictions, the audiologists and speech-language pathologists are resuming their services. The findings of the study are important as they help to highlight the areas that need more awareness among these professionals about appropriate and standard infection control measures. There is a need to have in place standard operating protocols and curriculum that includes importance of hand wash and infection control in Indian context. The findings might differ if obtained professionals from different countries, due to the differences in guidelines and outcomes of the pandemic. Similar studies among the same professionals from other countries could help in understanding the global perspective towards this outbreak and serve as a ready reckoner for future course of action.

\section{Declaration of competing interest}

There is no conflict of interest to disclose.

\section{Acknowledgement}

The authors thanks all the fellow professionals for participating in this study. We thank the Indian Speech-Language and Hearing Association for their support.

\section{References}

1 Dong E, Du H, Gardner L. An interactive web-based dashboard to track COVID-19 in real time. Lancet Infect Dis. 2020;3099(20):19-20. https://doi.org/10.1016/S14733099(20)30120-1.

2 World Health Organization. Coronavirus Disease (COVID-19) Advice for the Public.

3 World Health Organization. reportCoronavirus Disease (COVID-19) India Situation Report.

4 Ministry of Health and Family Welfare Government of India. Advisory on Social Distancing Measure in View of Spread of COVID-19 Disease.

5 World Health Organization. Coronavirus Disease (COVID-19) Outbreak: Rights,roles and Responsibilities of Health Workers, Including Key Considerations for Occupational Safety and Health.

6 Centers for Disease Control and Prevention. Coronavirus (COVID-19): Information for Healthcare Professionals. 
7 Ministry of Health and Family Welfare Government of India. Detail Question and Answers on COVID-19 for Public.

8 Academy of Doctors of Audiology. COVID-19 Resources.

9 American Speech-Language-Hearing Association. Infection Control Resources for Audiologists and Speech-Language Pathologists.

10 British Academy of Audiology. British Academy of Audiology Response to Covid-19: NHS England Essential Activity for Audiology.

11 World Medical Association. World medical association declaration of Helsinki: ethical principles for medical research involving human subjects. $J$ Am Med Assoc. 2013;310(20):2191-2194. https://doi.org/10.1001/jama.2013.281053.
12 Rowden-Racette K. Where the boys aren't. 18. The ASHA Leader; 2013:46-51. https:// doi.org/10.1044/leader.FTR1.18082013.46 (8).

13 World Health Organization. WHO Guidelines on Hand Hygiene in Health Care. 2009.

14 World Health Organization. reportNovel Coronavirus Disease (COVID-19) India Situation Report-7.

15 Centers for Disease Control and Prevention. Coronavirus Disease 2019 Manage Anxiety \& Stress.

16 Handwashing Liaison Group. Hand washing. Br Med J. 1999;318(7185):686. https:// doi.org/10.1007/bf02737760. 